\title{
Existence of vigorous lineages of crop-wild hybrids in Lettuce under field conditions
}

\author{
Danny A.P. HoOfTMAN ${ }^{1,2 *}$, Yorike HARTMAN ${ }^{1}$, J. Gerard B. OOSTERMEIJER ${ }^{1}$ and Hans (J.) C.M. Den NiJs ${ }^{1}$ \\ 1 Institute for Biodiversity and Ecosystem Dynamics, University of Amsterdam, The Netherlands \\ 2 NERC Centre for Ecology and Hydrology, Wallingford, UK
}

Plant to plant gene flow is a route of environmental exposure for GM plants specifically since crosses with wild relatives could lead to the formation of more vigorous hybrids, which could increase the rate of introgression and the environmental impact. Here, we test the first step in the process of potential transgene introgression: whether hybrid vigor can be inherited to the next generation, which could lead to fixation of altered, i.e., elevated, quantitative traits.

The potential for a permanent elevated fitness was tested using individual autogamous progeny lineages of hybrids between the crop Lactuca sativa (Lettuce) and the wild species Lactuca serriola (Prickly Lettuce). We compared progeny from motherplants grown under either greenhouse or field conditions. The survival of young plants depended strongly on maternal environment. Furthermore, we observed that offspring reproductive fitness components were correlated with maternal fitness.

Our study demonstrates that post-zygotic genotypic sorting at the young plants stage reduces the number of genotypes non-randomly, leading to inheritance of high levels of reproductive traits in the surviving hybrid lineages, compared to the pure wild relatives.

Consequently, directional selection could lead to displacement of the pure wild relative and fixation of more vigorous genome segments originating from crops, stabilizing plant traits at elevated levels. Such information can be used to indentify segments which are less likely to introgress into wild relative populations as a target for transgene insertion.

Keywords: Lettuce / vigorous-lineages / crop-wild hybrids / gene introgression

\section{INTRODUCTION}

Cultivated plants frequently occur sympatrically with their ancestral wild relatives, with which they are often interfertile, resulting in cross-pollination and hybridization (Chapman and Burke, 2006; Ellstrand, 2003; Hails and Morley, 2005). Newly introduced genes escaping from crops could confer competitive advantages to hybrid plants resulting in fixation, i.e. introgression of genomic segments originating from the crop into populations. In some cases these segments may alter quantitative traits of plants so that the ecology of whole plant populations could change (Chapman and Burke, 2006; Snow et al., 2005). Over the last two decades, introgression of GM cultivar genes into wild relative populations has become a cause for concern with respect to the impact of the genetic modifications on wild plant populations.

The ability of crops and wild relatives to hybridize is now generally accepted for most major crops (Andow

* Corresponding author: dann1@ ceh.ac.uk and Zwahlen, 2006; Chandler and Dunwell, 2008). Until recently, most of the resulting hybrids were considered inferior to the pure wild relative, as most crop genes are supposedly poorly suited to survival outside agricultural environments (Hails and Morley, 2005), making introgression unlikely. Although this may be true in many cases, there are various empirical examples of hybrids superior to their parents, i.e. showing hybrid vigor (Burke and Arnold, 2001; Ellstrand, 2003). In addition, there is accumulating evidence that a proportion of the hybrid genotypes can locally displace parental taxa (Ayres et al., 2004; Hegde et al., 2006; Milne and Abbott, 2000).

Hybrid vigor is generally caused by a combination of heterosis and novel epistatic interactions in recombinant genotypes (Burke and Arnold, 2001). Many known cases of hybrids being superior to wild relatives concern niche differentiation, such as in saltier or wetter habitats (i.e., Campbell and Snow 2007; Kirk et al., 2005; Rieseberg et al., 2003). A few studies have been carried out within typical habitats of the wild relative, examples are 
Campbell et al. (2005), Erickson and Fenster (2006), and Hooftman et al. (2005, 2007a). In these cases, the competitive ability of hybrids in a specific habitat has increased to such an extent that the "pure" species can locally be displaced (Damgaard and Jensen, 2002; Hooftman et al., 2007a). An important prerequisite is the addition of novel genetic variation to an existing gene pool on which selection can act (Ellstrand and Schierenbeck, 2006). Genes escaping from crops could provide this variation, resulting in a pool of recombinant genotypes with a potentially wider range of phenotypes. In a stressful, i.e. high mortality, environment survival becomes skewed among genotypes favoring the most advantageous traits for that environment. We will refer to this process as "genotypic sorting". As a consequence, selected plants would contain multiple crop-originating genome segments with a higher quantitative trait association, compared to their wild counterparts. When hybrid vigor remains consistent over multiple generations sorting could lead to fixation and successful introgression of those genomic segments. Therefore, identification of such sorting processes at the lineage level is the first step in identifying crop segments which could be under positive or negative selection (Chapman and Burke, 2006; Chapman et al., 2008; Stewart et al., 2003). Employing marker assisted breeding, this information could be used to target transgene insertion sites to those genomic regions providing the highest likelihood of being purged after escape into wild relatives populations (Stewart et al., 2003).

From the introduction above, we derive three main requirements for stabilizing elevated fitness and competitive ability of hybrids, compared to their wild parent: (i) some hybrid lineages should have superior fitness over the parental species during multiple generations; (ii) some traits should be genotypically selected to allow lineage sorting, which could lead to fixation; and (iii) the hybrid vigor needs to be inherited by part of the progeny. This balance between selection and inheritance is the focus of this paper as a first step to developing an assessment tool, including, and using, skewed inheritance through sorting. This is tested, employing a wellestablished hybrid system.

Hybrids between crop Lettuce (Lactuca sativa) and its fully interfertile wild relative $L$. serriola are fitter than their parents in selective environments, showing better survival (Hooftman et al., 2005, 2007a) and higher short-term resilience to pathogen attack (Hooftman et al., 2007b). These results suggest that the recent rapid spread of L. serriola in Europe (D'Andrea et al., 2009) could be partly associated with introgression of crop segments into the wild relative (Hooftman et al., 2006).

We use this Lactuca model system to identify directional patterns in phenotypic variation that indicate selection through sorting. In this system, requirement (i) of the list above is clearly met (Hooftman et al., 2005, 2007a, 2007b). Hence, the objectives of this study are to test the two remaining requirements:

(1) Identify whether genotypic sorting occurs in Lactuca hybrids, and in which traits, by comparing the progeny of motherplants grown in a previous experiment under high stress (= high mortality) semi-natural field conditions ("field-derived progeny") to the progeny of motherplants grown under low stress (= low mortality) greenhouse conditions ("greenhouse-derived progeny"). Where genotypic sorting has not altered the average phenotype among progenies, we expect no differences between both progenies. To avoid putative effects of local adaptation to soil and micro-climate, the $L$. serriola genotypes that were used had been collected in 2001 at least $250 \mathrm{~km}$ away from the semi-natural experimental field site.

(2) Test for the inheritance of reproductive fitness levels of maternal plants in their progeny when grown under similar conditions in the next year. This inheritance would be supported by a significant mother-offspring correlation. To test this, we selected progeny lineages originating from maternal plants with relatively low and relatively high total seed mass, from a previous field experiment (Hooftman et al., 2007a) and planted them into the same field.

In the previous field experiment in 2004, from which progeny lineages were collected, only $10-15 \%$ of plants survived to seed-set (Hooftman et al., 2007a). Hence, there was a large window of opportunity for rapid selection through sorting, if many less adapted recombinant genotypes were purged from the population. We tested for this in a study, conducted in 2005. For both objectives we distinguished between hybrids that were either only allowed to self-fertilize after the $\mathrm{F}_{1}$ creation ("selffertilization pathway"), or in which the genomic content of the crop was diluted by backcrossing to the wild parent ("backcross pathway").

\section{RESULTS}

\section{Effects of progeny source}

"Field-derived progeny" survived better in the first month than "greenhouse-derived" progeny for all classes (Tab. 1; Fig. 1a). Moreover, the coefficient of variation for survival in the first month among individual plants within lineages was also lower for field-derived than for greenhouse-derived progeny (Tab. 2). The combination of both effects suggests a narrower, better-adapted set of genotypes in field-derived progeny, indicative of strong sorting during the previous year in the field. No other general patterns were present explaining within-lineage 
Vigorous lineages in Lettuce

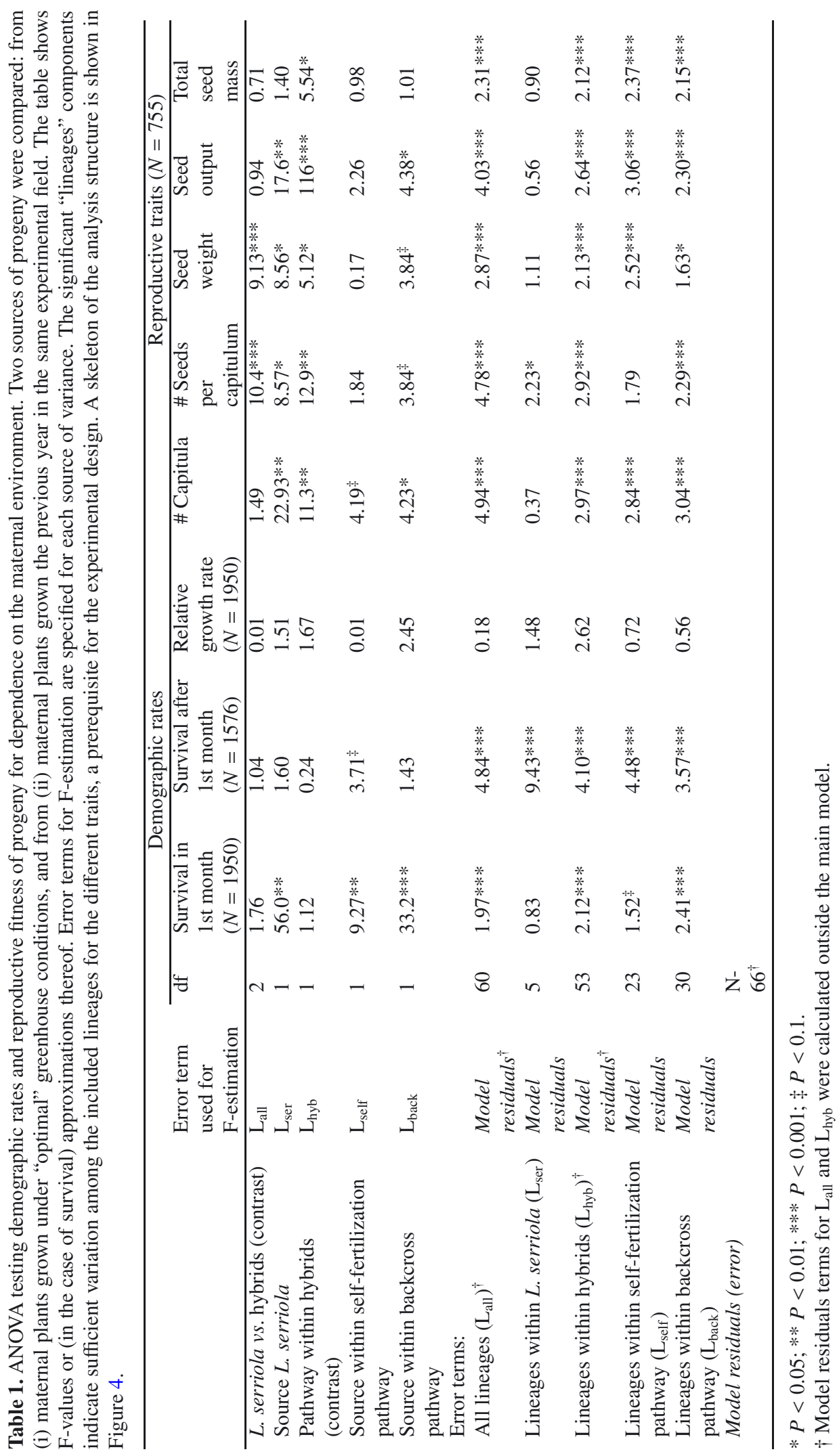



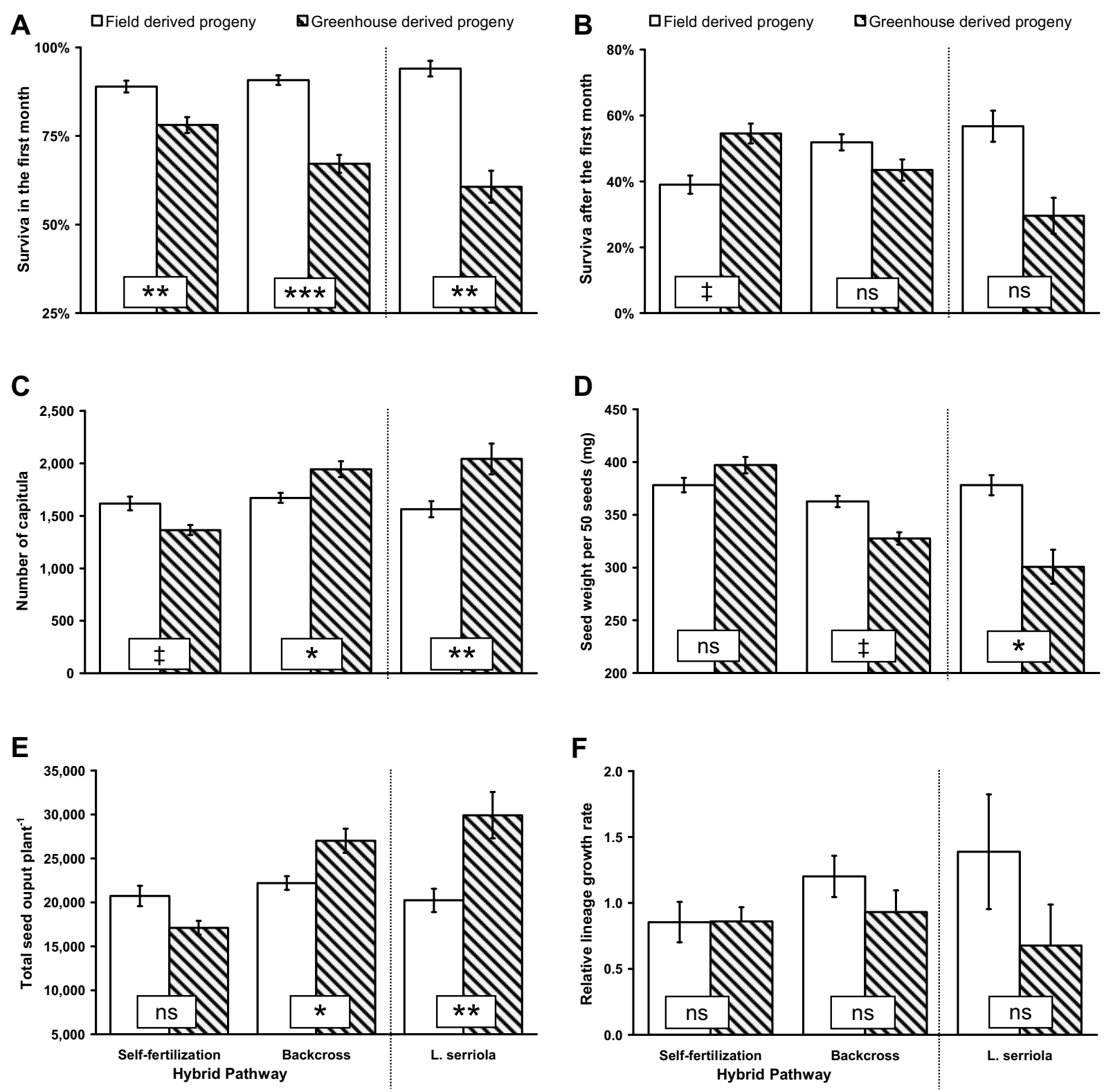

Figure 1. Comparison of planted progeny from (i) maternal plants grown under optimal greenhouse conditions, and (ii) maternal plants grown under putatively selective semi-natural field conditions: A: Survival of plants to the first month after planting (\%); B: survival after the first month until seed set (\%). Reproductive traits of individual plants: C: Number of capitula; D: seed weight (mg) per batch of 50 seeds; E: total seed output; and F: the relative population growth rate per lineage. Given are class averages \pm SEM. Significance levels between sources within categories are provided $(* P<0.05 ; * * P<0.01 ; * * * P<0.001, \ddagger P<0.1)$.

variation between greenhouse and field, with the single exception of seed weight in the self-fertilization pathway (Tabs. 1 and 2; Fig. 1).

For fitness traits in the self-fertilization pathway, we did not find a general effect of progeny origin on plant phenotype, with marginal exceptions for survival after the first month and the number of capitula $(P=0.07$ and 0.05 respectively; Fig. 1; Tab. 1). In contrast, in the backcross pathway, greenhouse-derived progeny had significantly more capitula, which resulted in a higher total seed output compared to field derived progeny. In addition, the average number of seeds per capitulum was significantly higher and the individual seed weight was significantly lower in the greenhouse derived progeny (both 
Vigorous lineages in Lettuce

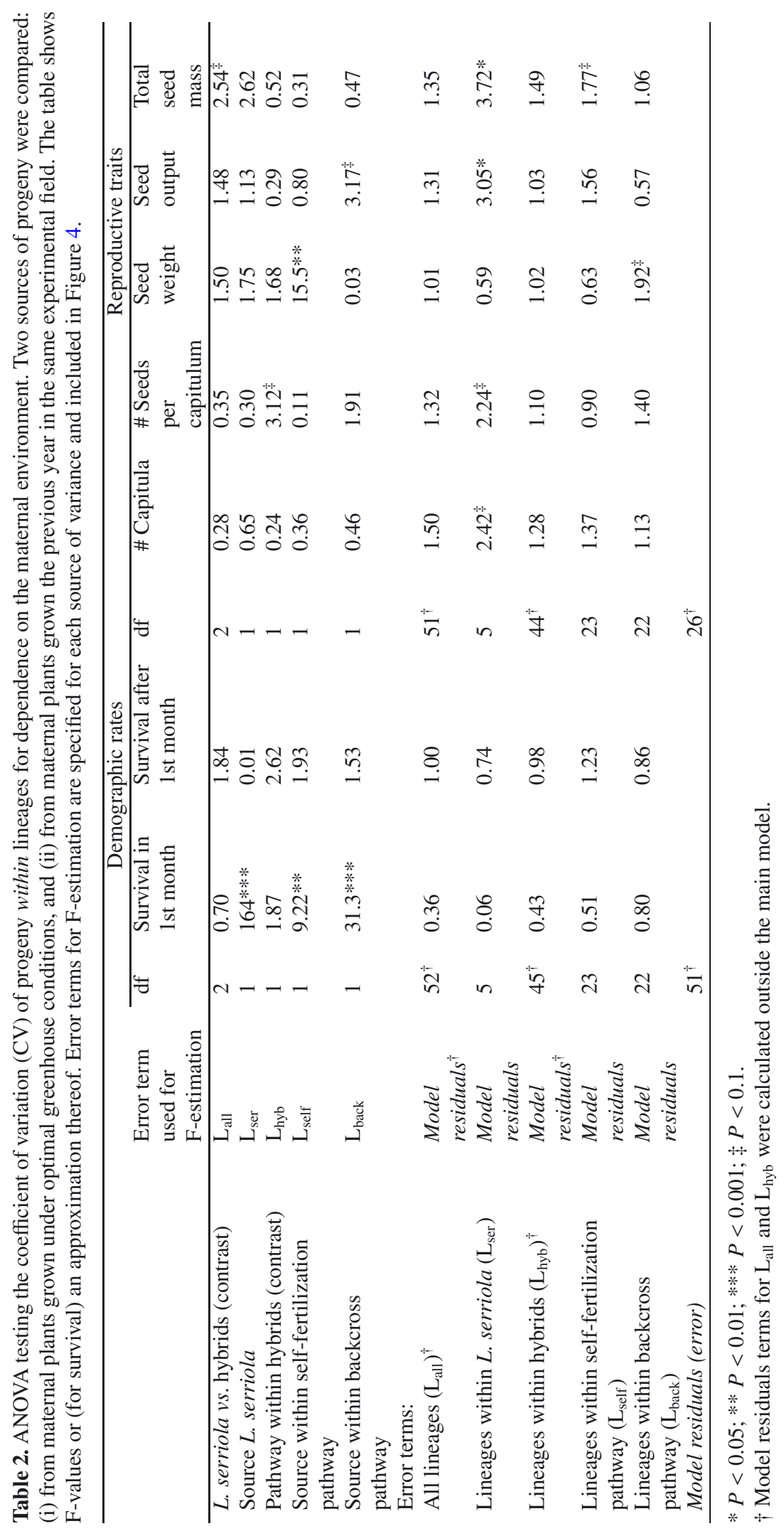


$P=0.06)$. Since the effects on total seed output and seed weight were opposite, no difference in total seed mass and relative growth rate per lineage was found between both sources (Tab. 1; Fig. 1). These results support a seed number/seed weight trade-off providing the potential for fixation of genome segments of different parental species.

These effects were observed in all individual classes of backcrossed hybrids $\left(\mathrm{BC}_{1}, \mathrm{BC}_{1 \mathrm{~S} 1}, \mathrm{BC}_{2}\right.$ and $\mathrm{BC}_{3}$; data not shown). Notably, in this respect, the backcross pathway behaved similar to $L$. serriola, suggesting that the potential for field-based selection is caused by an increased proportion of $L$. serriola genes in the genome.

\section{Inheritance of maternal fitness levels}

Reproductive fitness trait levels of hybrid progeny correlated significantly with that of their mother in both hybrid pathways (Tab. 3, Fig. 2). Hence, trait levels from the motherplants seem partially inherited. For $F_{1}$ hybrids this was not the case, except for seed weight. From these results we conclude that maternal plants with higher reproductive trait levels were more likely to produce progeny of which also had higher trait levels. This was true at least from the second hybrid generation onwards.

The demographic vital rates were inconclusive with respect to inheritance, as was the consequential relative lineage growth rate. In the case of survival in the first month, this could be caused by the fact that survival in 2005 was much higher (85-90\%) than in the previous experiment in $2004(10-15 \%)$.

Since these experiments were done (i) in outdoors, i.e., non-greenhouse conditions, and (ii) in segregating hybrid populations, the between-year correlations used as proxy for $\mathrm{H}^{2}$ were generally low $(<50 \%)$. Moreover, the observed values for reproductive traits were generally higher for motherplants in 2004 than for their progeny in 2005. Since the field site and experimental set-up were the same for both experiments, we attribute these differences to unexplained between-year variation.

\section{DISCUSSION}

Directional genotypic sorting among Lactuca hybrid genotypes is plausible; the signal already seems to be detectable after one year, suggesting that selection is strong. We base this on observations from transplant experiments within the core niche of the wild species, $L$. serriola. We found a narrowed set of genotypes in progeny from motherplants exposed to high stress field conditions compared to offspring formed under low stress greenhouse conditions. Compared to the latter group, the "fieldderived" progeny lineages had both a higher survival rate in the first month and reduced phenotypic variation when planted in a semi-natural field resembling the habitat of the wild species.

Strikingly, for reproductive fitness traits, the backcross pathway ( $\leq 25 \%$ crop genome) showed a clear signal of genotypic sorting which was absent in the selffertilization pathway ( $\approx 50 \%$ crop genome). The backcross pathway followed similar patterns as the wild species, $L$. serriola, both in direction and significance of effects. The potential for selection is therefore most likely associated with the L. serriola part of the genome. At our field site, sorting within L. serriola was possible because the employed genotypes originated from different soil types and hence were not locally adapted. With a higher $L$. serriola content in backcrossed hybrids, similar genotypes could have been favored. Furthermore, hybrids might have become more adapted to growth in noncontrolled environments because backcrossing increased genomic stabilization.

\section{Alternative explanations}

Several alternative explanations for our results can be formulated. First, short-term, environmentally induced, maternal effects (e.g., Galloway, 2005), for instance brought about by the richer soil of the field site, could have resulted in field-derived progeny with a higher seed mass and so better chances of early survival. Second, local adaptation may have been caused by tailoring gene expression patterns in response to the local environment (i.e. epigenetic effects), rather than reflecting adaptive genetic variation (Rapp and Wendel, 2005). However, both alternatives seem less likely since the direction of the observed patterns would have been consistent for all classes of plants.

In addition, local adaptation of the source population to this specific environment is not likely. Between the experimental field site and the populations from which the L. serriola genotypes were collected, the distance is at least $250 \mathrm{~km}$ and there was a large difference in soil type. Source population Eys occurs on loess and Zarrentin on sand, whereas the experimental field was established on former agricultural, nutrient-rich clay.

Based on the arguments above, we suggest that there has been genotypic sorting favoring the genotypes best adapted to this local environment. Similar, environment induced, patterns have been found for genotypes as a whole (e.g., Campbell et al., 2008) or for selection of quantitative trait loci (QTLs; Baack et al., 2008).

\section{Fecundity inheritance}

The reproductive capacity of maternal plants seems to be inherited by at least part of their progeny. Maternal lineages with higher reproductive traits are thus more likely 
Vigorous lineages in Lettuce

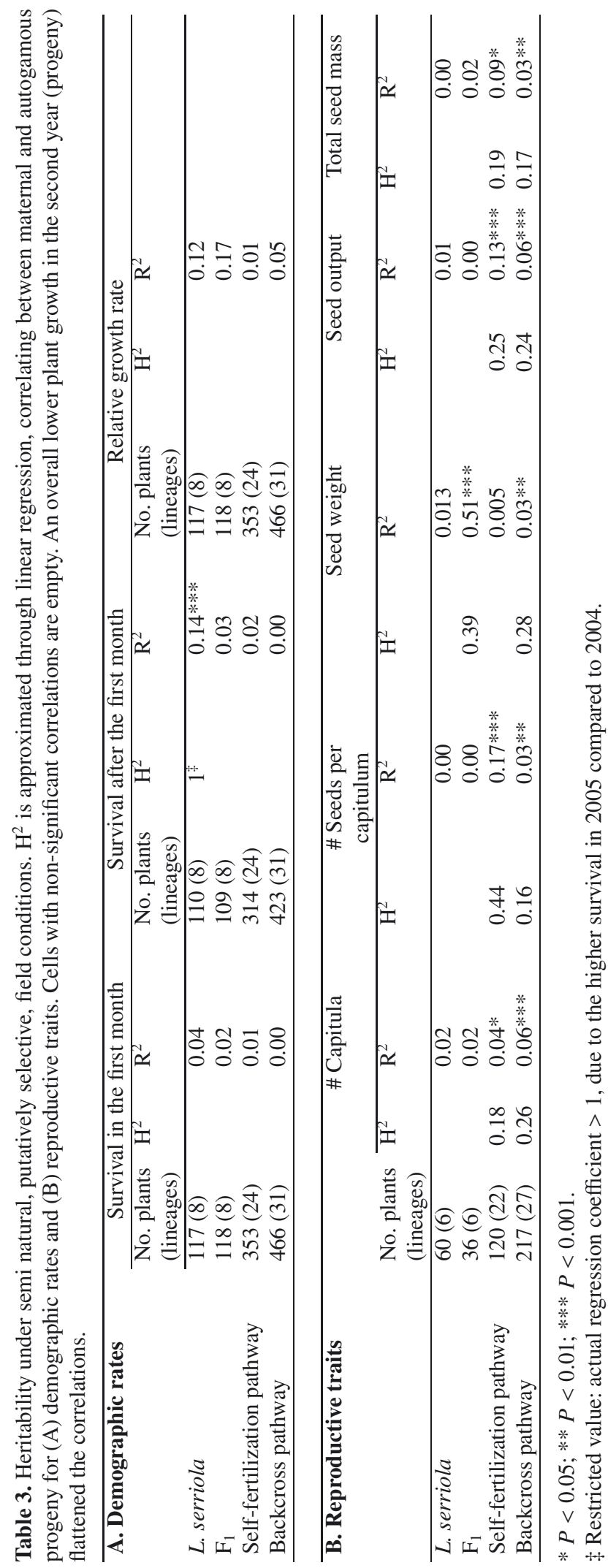



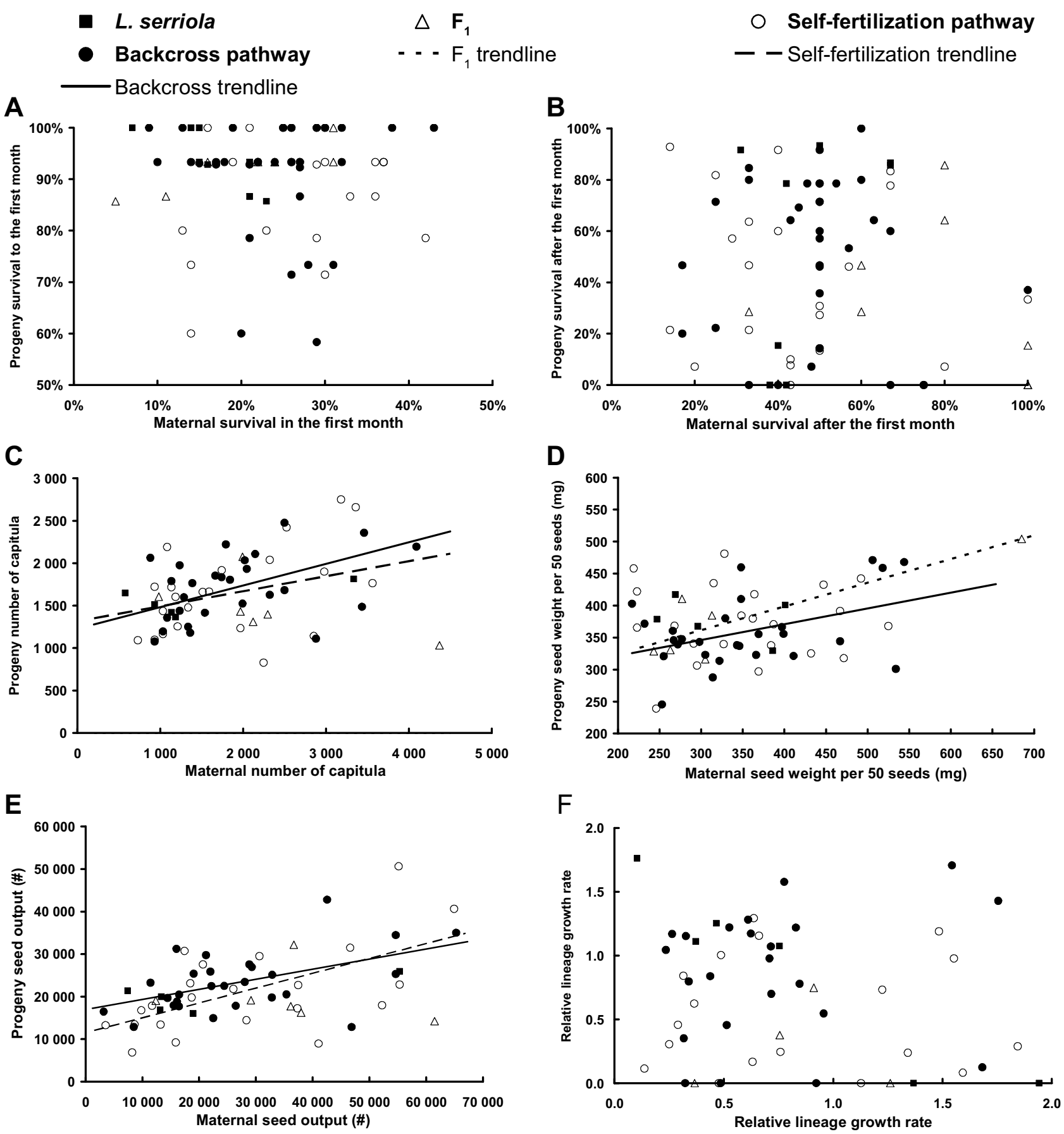

Figure 2. Correlation between maternal traits and progeny traits. Shown are averages per seed-lineage within the different categories (L. serriola, $\mathrm{F}_{1}$, self-fertilization- and backcrossing pathway); significant trendlines are depicted for hybrid categories. Demographic rates: A: Survival of plants to the first month after planting (\%); B: survival after the first month until seed set (\%). Reproductive traits of individual plants: C: Number of capitula; D: seed weight $(\mathrm{mg})$ per batch of 50 seeds; E: total seed output; and F: the relative population growth rate per lineage. Note that the statistical analysis was done at the individual plant level but, for convenience, averages per lineage are shown in the figures. 
to return a higher fraction of high-trait level progeny. Still, the variation in trait values was large for all classes, probably as a combined result of segregation and stochastic effects on plant growth.

In 2004 , when only $10-15 \%$ survived until seed set, selection in the maternal plants was strong (Hooftman et al., 2007a). It is therefore likely that only a narrow, better adapted set of genotypes survived these field conditions. If this selection continued over multiple generations, consistently sorted "high-fitness" lineages could gain dominance in a hybrid population. This is because they are either better direct competitors (Damgaard and Jensen, 2002) or gradually displace other lineages by producing more offspring (Hall et al., 2006). Moreover, the breakdown of hybrid vigor by recombination could become skewed by a strong sorting of specific genotypes (Brateler et al., 2006). Consequently, large genome segments of one of the parental species could become fixed, stabilizing alterations in plant performance and morphology (Burke and Arnold, 2001). Therefore, these results are the first steps in linking quantitative traits under selection, to the likelihood of introgression into wild relatives of crop segments associated to these traits.

The bottleneck for survival occurred in young plants. High mortality in at least one life phase is necessary to allow sorting of the better locally adapted recombinant genotypes. However, at a trait level, selection was not necessarily in concert towards one of the parental species, because "greenhouse-derived" offspring produced more, albeit lighter, seeds than "field-derived" progeny. This suggests selection could provide a mosaic of segments of both species in the more successful genotypes. Preliminary molecular evidence supports this hypothesis. In plants used for the 2003 field experiments at the same site (Hooftman et al., 2005), we found a mosaic of distorted chromosomal segments, originating from both parental species, in these hybrids in the field (Orozco-ter Wengel et al., 2005). Similar genomic mixtures were found in Helianthus (Baack et al., 2008; Dechaine et al., 2009), Raphanus (Campbell et al., 2009) and Oryza (Grillo et al., 2009).

Importantly, sorting based on quantitative traits assumes a stable environment. Here, we did not test whether temporal stochasticity within a single environment or performance of genotypes in different habitats would lead to $G \times E$ interactions. Mercer et al. (2007) and Vuilleumier et al. (2008) further discuss such interactions.

\section{Hybrid vigor and displacement potential}

In earlier studies we suggested that at least part of the crop genome could introgress into the genomic background of $L$. serriola. A strong hybrid vigor was found for four generations in hybrid lineages, putatively because of formation of novel additive combinations (Hooftman et al., 2005, 2007a, 2007b). Such new combinations could quickly spread in a population (Hooftman et al., 2008; Huxel, 1999).

An important caveat in many study designs is the differentiation between analyzing the fitness of individual lineages and the average fitness of a class of hybrid plants (e.g., based on crop genome percentage). The latter is likely to contain many genotypes. Through the combination of sorting and inheritance one vigorous genotype could become dominant, out-competing other - less fit lineages. The average fitness across all lineages would consequently be an erroneous extrapolation of the potential of spread of hybrid taxa.

\section{Genotypic sorting within parental habitats}

Hybrids are often considered to have the potential to occupy ecological niches that differ from those of their parents. This ecological differentiation has been regarded as the major evolutionary force promoting expansion of hybrid populations (Burke and Arnold, 2001). Development of trait values absent in either of the parental species has been found in a range of taxa, which would explain the occupation of previously unsuitable habitats (e.g., Burgess and Husband, 2006; Campbell and Snow, 2007; Kirk et al., 2005; Rieseberg et al., 2003).

Whether hybrids could also outcompete parental populations in their natural habitat has received much less attention. However full displacement of resident taxa by hybrids in their own habitat has been observed to occur within decades (Hegde et al., 2006; Milne and Abbott, 2000). Experiments have shown that the fitness of more advanced hybrid generations could be equal or better than that of their parent(s) within the same habitat (Erickson and Fenster, 2006; Hooftman et al., 2007a; Ridley and Ellstrand, 2009; Snow et al., 2010). Our results provide further evidence that displacement of parental species by hybrids could be promoted by combined inheritance of quantitative reproductive trait levels and genotypic sorting in the earlier stages of plant growth. This process could lead to rapid fixation of genomic segments originating from both parental species, potentially providing beneficial epistatic interactions. Fixation could be rapid, especially when crop genes are introgressing into predominantly autogamous species and genetic reshuffling caused by recurrent outcrossing is occurring much more infrequently (Charlesworth et al., 2003).

L. sativa was presumably derived from $L$. serriola about 5000 years ago (Ryder, 1999) and is generally bred using worldwide collected $L$. serriola accessions. Consequently, it will mainly consist of a subset of genes from the broad gene pool of $L$. serriola. No genetic barriers 
between the species are known (Koopman et al., 2001). Most genes escaping from $L$. sativa would be fully compatible with $L$. serriola and are thus a potential source of new additive variation to local genotypes. This has two important implications. First, large scale maladaptation to a semi-natural habitat is less likely since most of the recombinant genotypes would remain within the actual niche of the wild species. Secondly, the variation added from the crop could allow for the formation of vigorous genotypes through swapping "wild" genes for locally better performing ones from the crops. However, which genes or traits allow for such elevated fitness would be environment specific (Moles et al., 2008).

In summary, we found not only indications that fitness of hybrids could be higher, as concluded in earlier studies (Hooftman et al., 2005, 2007a), but also that it might stabilize and thus result in introgression of crop originating genome segments. Therefore, to assess the biosafety of genetically modified crops, it seem necessary to debate the inclusion of measures testing whether and in what quantity such vigorous, stabilized, hybrid lineages could occur.

\section{Implication and future prospects}

Returning to the main requirements for elevated competitive ability that we put forward in the introduction, the fitness of hybrids is higher than that of the pure wild relative for multiple generations (requirement $\mathrm{i}$ ), as shown in earlier studies (Hooftman et al., 2005, 2007a, 2007b). In this study we found indications for genotypic sorting (req. ii). Moreover, this hybrid vigor seems to be partly inherited in progeny lineages (req. iii). Linking these fitness alterations to the current rapid spread of $L$. serriola throughout Europe is tempting (D'Andrea et al., 2009; Hooftman et al., 2006), also since $F_{1}$ hybrids or selfings thereof have been reported. However, investigations into a possible direct molecular link between introgression and the spread are still ongoing. Nonetheless, based on the combined analysis of our synthetic hybrid lineages, introgression seems likely, now or in the future.

The main message of this paper is that a genetic basis for consistently vigorous hybrid genotypes appears to be present in Lactuca. We extrapolated this from one year of observations after high mortality under semi-natural field conditions. High fitness could become fixed in introgressant populations in cases where sorting within autogamous lineages consistently favors similar genotypes. However, survival capacity selected for is not necessarily linked to fecundity, as shown. Furthermore, the estimated levels of convergence in terms of level of trait values cannot be obtained from such short time frames and requires longer term studies.
Although our study focuses on the overall genome level rather than on specific genes, the aforementioned hybridization patterns are strongly related to the likelihood of introgression of genetic modifications (transgenes) via two mechanisms: (a) the transgene is part of the complementary package of interacting genes causing hybrid vigor (Ellstrand, 2003). (b) More likely, the transgene would not affect plant fitness itself but is in linkage disequilibrium with genes or gene combinations providing a selective advantage to plants, enabling introgression through genetic hitchhiking (Stewart et al., 2003).

Studies like this one, together with others (e.g. Baack et al., 2008), are the necessary first steps in identifying genomic segments which are more likely to introgress into wild relatives based on their trait associations. QTL approaches seem to be the most appropriate way forward (Collard et al., 2005; Heffner et al., 2009), employing the vastly expanding number of genetic maps (Collard and MacKill, 2008). Modeling studies have already shown that segments with only limited fitness advantage in the hybrid plants can provide rapid fixation of introduced genes (Hooftman et al., 2008; Huxel, 1999). Where recombination between a transgene and beneficial QTLs is low, introgression of such a gene is either facilitated or mitigated depending on the trait associations of the segment (Stewart et al., 2003; Sweet, 2009).

\section{MATERIALS AND METHODS}

\section{Taxa studied}

For extensive descriptions of the two parental species we refer to Flora Europaea (Tutin et al., 1976). In summary, Lactuca serriola L. (Asteraceae, $2 n=18$ ) is a common annual weed found in anthropogenically disturbed habitats, such as roadsides, railways and ruderal sites in urban areas throughout Europe, Northern Africa and North America. Recent changes in its distribution and ecology in The Netherlands have been described in Hooftman et al. (2006). Europe-wide changes in distribution are depicted in D'Andrea et al. (2009). Plants are predominantly autogamous with outcrossing rates through insect pollen vectors of approx. 1-5\% (D'Andrea et al., 2008).

Lactuca sativa L. (Asteraceae, lettuce, $2 n=18$ ) is a common annual crop. Left-over plants flower frequently in allotments, largely simultaneously and often sympatrically with $L$. serriola. $L$. sativa is considered to be derived from L. serriola (Ryder, 1999). Moreover, both taxa are fully interfertile, with no known pre- or post-zygotic barriers (Koopman et al., 2001). The outcrossing rate of L. sativa (0.5\%; Giannino et al., 2008) is similar to that reported for $L$. serriola. 


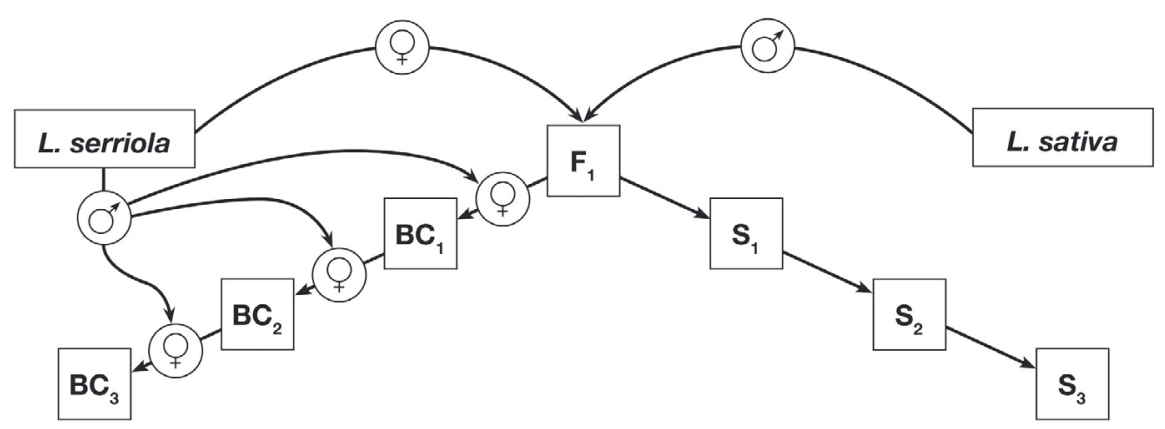

Figure 3. Pedigree of hybrid classes as generated under greenhouse conditions. $S_{1}, S_{2}$ and $S_{3}$ represent the self-fertilization pathway (all $\approx 50 \%$ crop type alleles), $\mathrm{BC}_{1}, \mathrm{BC}_{2}$ and $\mathrm{BC}_{3}$ the backcross pathway ( $\leq 25 \%$ crop type alleles).

\section{Plant material}

For details about the crossing scheme and protocols we refer to Hooftman et al. (2005). The crossing scheme included two cultivars (cv. Dynamite and cv. Meikoningin) and two $L$. serriola populations. The cultivars were considered to be genetically monomorphic. For $L$. serriola, seeds were collected in August 2001 from two spatially well-separated wild populations, i.e. from Eys, The Netherlands (N 50 49'; E $05^{\circ} 55^{\prime}$ ) and Zarrentin, Germany ( $\mathrm{N} \mathrm{53} 3^{\circ} 32^{\prime}$; E $10^{\circ} 54^{\prime}$ ). The first population is semi-permanent at the margin of an agricultural field on loess soil, known for over 20 years to the authors. The second population is highly transient in a sand quarry. Within each population, we collected seeds from 10 randomly sampled plants, providing a maximum of 40 "lines" per generation, which were kept separate throughout the whole crossing scheme. No broad-scale phenotypic differences attributable to site $\times$ cultivar interactions were identified, although we purposely included genotypic variation this way (Hooftman et al., 2005, 2007a).

- We created $\mathrm{F}_{1}$-progeny for all 40 lines using $L$. serriola as the maternal, and $L$. sativa as the paternal parent, thus simulating crop to wild gene flow (Fig. 3). $F_{1}$ 's were genetically verified in the context of the study by Hooftman et al. (2005).

- Backcrossing was done by using hybrids maternally and $L$. serriola paternally, mimicking the most likely first steps in the introgression process of crop genes into wild relative populations. We created three generations of backcrossed hybrids: $\mathrm{BC}_{1}$ (assumed $25 \%$ average crop genome), $\mathrm{BC}_{2}(12.5 \%)$ and $\mathrm{BC}_{3}$ $(6.25 \%)$. The selfed cohort of the $\mathrm{BC}_{1}$ generation, $\mathrm{BC}_{1 \mathrm{~S} 1}$, is included as well. These joint $\mathrm{BC}_{\mathrm{x}}$ generations are referred to as "backcross pathway".

- For establishing 2nd to 4th generation hybrids along the "self-fertilization pathway", we let $\mathrm{F}_{1}$-hybrids self-pollinate to obtain a $S_{1}$ generation, subsequent selfing led to the $S_{2}$ and $S_{3}$ generations (all with on average $50 \%$ crop genome).

The entire crossing pedigree is shown in Figure 3. To obtain the necessary statistical power and derive conceptual simplicity, we investigated per pedigree pathways in this paper. Consequently we pooled autogamous progeny of $S_{1}, S_{2}$ and $S_{3}$ generations (average $50 \%$ crop genome) along the self-fertilization pathway and $\mathrm{BC}_{1}$, $\mathrm{BC}_{1 \mathrm{~S} 1}, \mathrm{BC}_{2}$ and $\mathrm{BC}_{3}$ generations along the backcross pathway ( $\leq 25 \%$ crop genome). The $\mathrm{F}_{1}$ generation and the pure wild relative, $L$. serriola, were treated separately.

\section{Experimental set-up}

The experimental set-up was split into two parts: (i) A prior experiment performed in 2004 in which the motherplants were grown under either high mortality field or non-selective greenhouse conditions, and (ii) a new experiment in 2005 in which the progenies of these motherplants were tested under field conditions. This paper describes the results of the new 2005 experiment; the results of the previous 2004 experiment have been published in Hooftman et al. (2007a), accordingly only specific details about the design are provided below.

\section{The 2004 experiment}

In this experiment we tested the fitness of eight different classes of hybrids at three different locations. Two $169 \mathrm{~m}^{2}$ plots per location were created, each divided in four blocks. In April 2004, we sowed 400 seeds individually per block in a grid consisting of $30 \times 30 \mathrm{~cm}$ cells. Each subplot contained a full sample of all lines, placed at fully randomized grid positions. Seed positions were individually labeled and background germination of Lactuca was removed during the experiment. A spontaneously growing ruderal pioneer vegetation was allowed to emerge excluding only $L$. serriola for practical reasons; exerting 
a competitive pressure similar to many natural habitats in which $L$. serriola occurs. The vegetation included typical ruderal species like Brassica rapa, Capsella bursapastoris, Chenopodium spp., Cirsium arvense, Dactylis glomerata, Holcus lanatus, Poa annua, Senecio vulgaris, Sonchus asper and Taraxacum officinale (list drawn in July 2009 in plots of further experiments at the same location and therefore indicative only).

This treatment caused $\pm 85-90 \%$ mortality from seed to seed set. We presume that this design - i.e., high competition intensity causing low survival rates - provided ample opportunity for genotypic sorting. Therefore, these progenies could be selected for traits beneficial under these semi-natural field conditions. All seeds employed in this 2004 experiment were previously collected from plants in the greenhouse, autogamous descendants of the individuals collected in 2001. Hence any local adaptation would be specific to the original habitat of the L. serriola lines and not to the field site used for experiments.

\section{The 2005 experiment}

From all individual surviving plants in the field ("motherplants") in the 2004 experiment, progeny was collected that was presumably derived through autogamy, assuming an outcrossing level of approx. $1 \%$. We will refer to these progeny as "field-derived" and to the separate progeny families per motherplant as "lineages", i.e., the term "lineage" refers to the offspring of a single plant in the 2004 experiment. Autogamous full-sibs of the plants in the 2004 experiment were grown in the greenhouse and their seeds collected. We refer to these latter progeny as "greenhouse-derived". These progeny lineages are not selected, i.e., all plants survived until seed set.

We selected from the 2004 experiment at one location and within every hybrid class and $L$. serriola, four motherplants exhibiting the highest fitness in the field and four with among the lowest fitness, irrespective of the line (see crossing design, Fig. 3). In this way, we maximized the range of variation per hybrid class. However, by doing so, the identical main-lines and lines are not necessarily included in both pathways. Hence, the interactions between lines and pathways cannot be estimated. In all analyses we did not group those lineages into binominal groups (high, low), but used their individual fitness, since they often represent a continuum rather than a sharp distinction. The lineage choice was restricted by seed availability for both field- and greenhouse derived progeny.

Our measure of fitness was the "total seed mass produced per motherplant". The advantage of this measure is that it is less sensitive to the trade-off between seed weight and total seed output seen in earlier studies (Hooftman et al., 2005, 2007a).

We used the experimental plots at our field location in Sijbekarspel, The Netherlands ( $52^{\circ} 42^{\prime}$; E $05^{\circ} 00^{\prime}$ ).
Two $169 \mathrm{~m}^{2}$ plots were demarcated and ploughed to mimic anthropogenic soil disturbance and to remove remnants of prior experiments, including the 2004 previous experiment. The experimental soil was well-drained clay, nutrient-rich from former agricultural use. Each plot was divided into four blocks; each block containing a grid of 400 planting points with a distance of $30 \mathrm{~cm}$ between plants. A pioneer vegetation was again allowed to emerge spontaneously which included species mentioned in the 2004 experiment. Seeds were germinated in jiffy ${ }^{\circledR}$-pots, plantlets were transplanted into the field at the first true leaf stage in May 2005. We used 15 seedlings per lineage, with one block containing three plants and the others, four. The plants were fully randomized among grid positions within blocks.

\section{Measurements}

We measured the survival rate in the first month, defined as "the likelihood of presence of a plant, irrespective of its life phase, one month after planting". The survival rate until seed set was determined as "the likelihood that a plant, which was present after one month, successfully developed into a reproductive adult". At the first stage of seed set we measured per plant: (i) the number of reproductive basal shoots and (ii) the number of branches on the main stem. For subsequent estimation of (iii) the number of capitula, we used the regression provided by Hooftman et al. (2005; eq. (1)). To assess (iv) seed production per capitulum, 10 randomly picked fruiting capitula per plant were sampled and the number of seeds counted. Subsequently, a batch of 50 seeds was weighed on a microbalance to obtain (v) the average seed weight. The total seed output (vi) was subsequently calculated by multiplying (iii) and (iv). Moreover, we multiplied (vi) with (v) to obtain the total seed mass per plant. To obtain the relative population growth rate per lineage, we multiplied (vi) with survival in the first month and to seed set. To obtain values relative to the pure wild relative, we divided the obtained numbers to the combined average of all L. serriola lineages and origins (Hooftman et al., 2007).

\section{Statistical analyses}

Prior to the analyses, we grouped the different classes of hybrids into classes representing a full autogamous origin after the initial cross (self-fertilization pathway) and backcrossing towards $L$. serriola (backcross pathway). $\mathrm{F}_{1}$ hybrids were kept as separate groups, being part of either pathway. Note that we included a range of maternal fitness as wide as possible, so that among lineage variation should be high and preferably significant in an ANOVA. 


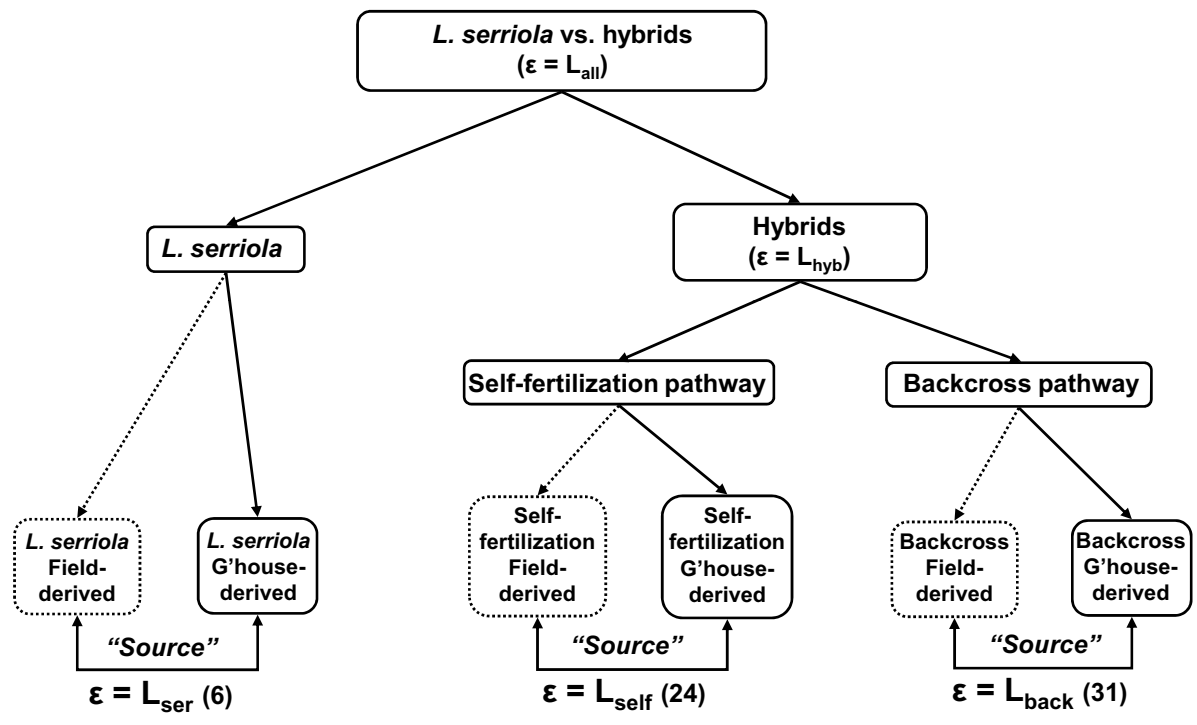

Figure 4. Skeleton of the testing procedure between sources (greenhouse and field derived progeny). Included are the error estimations $(\varepsilon)$ used for F-calculation in a Type I (hierarchical) ANOVA. L. serriola is distinguished from the hybrid classes by a linear contrast (L. serriola, vs. hybrids), allowing subsequent separate testing within these categories. Similarly, a contrast is used allowing subsequent separate testing within pathways. Errors are: $\mathrm{L}_{\text {all }}$ : all lineages including all categories; $\mathrm{L}_{\mathrm{hyb}}$ : lineages (lg') within all hybrids categories; $\mathrm{L}_{\mathrm{ser}}$ : $\lg$ ' within L. serriola; $\mathrm{L}_{\text {self }}$ : lg' within the self-fertilization pathway; $\mathrm{L}_{\text {back }}: \lg$ ' within the backcrossing pathway. $\mathrm{L}_{\text {all }}$ and $\mathrm{L}_{\text {hyb }}$ are calculated outside the main model, since they explain the same variation as $\mathrm{L}_{\text {ser }}, \mathrm{L}_{\text {self }}$ and $\mathrm{L}_{\text {back }}$. Provided is the number of lineages per pathway $(x)$.

Therefore, the used lineages do not necessarily give a reliable indication of the average fitness of a generation as a whole or the main lines within it. These fitness differences between classes of hybrids and compared to the wild relative were presented in earlier studies (Hooftman et al., 2005, 2007a).

To test for trait level differences between field and greenhouse-derived progeny, we used ANOVAs with sequential sum of squares (procedure GLM, SPSS statistical package, SPSS Inc.). For survival, we conducted an Analysis of Deviance (GENSTAT, Lawes Agricultural Trust, Harpenden, UK). We calculated ratios of mean deviance changes (quasi F-values), which approximately follow the F-distribution.

The skeleton of this statistical analysis is shown in Figure 4. To test differences between $L$. serriola and all other lineages we used a linear contrast ( $L$. serriola, $v s$. hybrids), using the hierarchic structure of the model to enable subsequent separate testing within $L$. serriola and specific hybrid categories. Subsequently, a similar linear contrast was used to distinguish between the self-fertilization and backcrossing pathways. We tested for source (i.e., field or greenhouse) dependence of the progeny lineages within the three categories; the included number of lineages per category is also shown in Figure 4. The rationale behind this design is that it is similar to separate testing per category in different tests. However, this also provides the opportunity to test between-categories at higher levels (i.e., hybrids $v s$. wild type and self-fertilization $v s$. backcrossing pathway), as well as allowing for scaling of the marginal effects among categories. The different categories are statistically independent. Nevertheless, they can be compared for the direction and strength of the effects, having been subjected to the identical selective force.

Changes in phenotypic range between the sources within lineages were tested using the coefficient of variation (CV) among all individuals within each lineage.

To test the dependence of reproductive traits and survival rates on maternal fitness we performed a linear regression per category. A significant linear regression coefficient is regarded as a proxy for broad-sense heritability, $\mathrm{H}^{2}$ (Lynch and Walsh, 1998). We restricted $\mathrm{H}^{2}$ between $0-1$.

\section{ACKNOWLEDGEMENTS}

We thank Maaike de Jong, Katja Mamonova, Jacqueline Kummer, and the technical staff of IBED for practical support. Eric Schranz, Bao-Rong Lu, Sophie Karrenberg, Stefan Andersson and two anonymous reviewers are acknowledged for their useful comments on earlier versions 
of this manuscript, Jan van Arkel for help with the figures. This study was supported by EU-QLK3-2001-01657.

Received February 13, 2010; accepted June 20, 2010.

\section{REFERENCES}

Andow DA, Zwahlen C (2006) Assessing environmental risks of transgenic plants. Ecol. Lett. 9: 196-214

Ayres DR, Smith DL, Zaremba K, Klohr S, Strong DR (2004) Spread of exotic cordgrass and hybrids (Spartina sp.) in the tidal marshes of San Francisco bay, California. Biol. Invas. 6: 221-231

Baack EJ, Sapir Y, Chapman MA, Burke JM, Rieseberg LH (2008) Selection on domestication traits and quantitative trait loci in crop-wild sunflower hybrids. Mol. Ecol. 17: 666-677

Brateler M, Lexer C, Widmer A (2006) Genetic architecture of traits associated with serpentine adaptation of Silene vulgaris. J. Evolution. Biol. 19: 1149-1156

Burgess KS, Husband BC (2006) Habitat differentiation and the ecological costs of hybridization: the effects of introduced mulberry (Morus alba) on a native congener (M. rubra). J. Ecol. 94: 1061-1069

Burke JM, Arnold ML (2001) Genetics and fitness of hybrids. Annu. Rev. Gen. 35: 31-52

Campbell DR, Galen C, Wu CA (2005) Ecophysiology of first and second generation hybrids in a natural plant hybrid zone. Oecologia 144: 214-225

Campbell DR, Waser NM, Aldridge G, Wu CA (2008) Lifetime fitness in two generations of Ipomopsis hybrids. Evolution 62: 2616-2627

Campbell LG, Snow AA (2007) Competition alters life history and increases the relative fecundity of crop-wild radish hybrids (Raphanus spp.). New Phytol. 173: 648-660

Campbell LG, Snow AA, Sweeney PM, Ketner JM (2009) Rapid evolution in crop-weed hybrids under artificial selection for divergent life histories. Evol. Appl. 2: 172-186

Chandler S, Dunwell JM (2008) Gene flow, risk assessment and the environmental release of transgenic plants. Crit. Rev. Plant Sci. 27: 25-49

Charlesworth B, Charlesworth D, Barton NH (2003) The effects of genetic and geographic structure on neutral variation. Annu. Rev. Ecol. Evol. 34: 99-125

Chapman MA, Burke JM (2006) Letting the gene out of the bottle: the population genetics of genetically modified crops. New Phytol. 170: 429-443

Chapman MA, Pashley CH, Wenzler J, Hvala J, Tang SX, Knapp SJ, Burke JM (2008) A genomic scan reveals candidates for genes involved in the evolution of cultivated sunflower. Plant Cell 20: 2931-2945

Collard BCY, MacKill DJ (2008) Marker-assisted selection: an approach for precision plant breeding in the twenty-first century. Philos. T. R. Soc. B. 363: 557-572
Collard BCY, Jahuzer MZZ, Brouwer JB, Pang ECK (2005) An introduction to markers, quantitative trait loci (QTL) mapping and marker assisted selection for crop improvement: the basic concepts. Euphytica 142: 169-196

D'Andrea L, Felber F, Guadagnuolo R (2008) Hybridization rates between lettuce (Lactuca sativa) and its wild relative (L. serriola) under field conditions. Environ. Biosafety Res. 7: 61-71

D'Andrea L, Broennimanm O, Kozlowski G, Guisan A, Morin X, Keller-Senften J, Felber F (2009) Climate change, anthropogenic disturbance and the northward range expansion of Lactuca serriola (Asteraceae). J. Biogeogr. 36: 1573-1587

Damgaard CJ, Jensen BD (2002) Disease resistance in Arabidopsis thaliana increases the competitive ability and the predicted probability of long-term ecological success under disease pressure. Oikos 98: 459-466

Dechaine JM, Burger JC, Chapman MA, Seiler GJ, Brunick R, Knapp SJ, Burke JM (2009) Fitness effects and genetic architecture of plant-herbivore interactions in sunflower cropwild hybrids. New Phytol. 184: 828-841

Ellstrand NC (2003) Dangerous liaisons? When cultivated plants mate with their wild relatives. Baltimore, John Hopkins University Press

Ellstrand NC, Schierenbeck KA (2006) Hybridisation as stimulus for the evolution of invasiveness in plants. Euphytica 148: $35-46$

Erickson DL, Fenster CB (2006) Intraspecific hybridization and the recovery of fitness in the native legume Chamaecrista fasciculata. Evolution 60: 225-233

Galloway LF (2005) Maternal effects provide phenotypic adaptation to local environmental conditions. New Phytol. 166: 93-99

Giannino D, Nicolodi C, Testone G, Di Giacomo E, Iannelli MA, Frugis G, Mariotti D (2008) Pollen mediated transgene flow in lettuce (Lactuca sativa L.). Plant Breed. 127: 308-314

Grillo MA, Li CB, Fowlkes AM, Briggeman TM, Zhou AL, Schemske DW, Sang T (2009) Genetic architecture for the adaptive origin of annual wild rice, Oryza nivara. Evolution 63: $870-883$

Hails RS, Morley K (2005) Genes invading new populations: a risk assessment perspective. Trends Ecol. Evol. 20: 245-252

Hall RJ, Hastings A, Ayres DR (2006) Explaining the explosion: modelling hybrid invasion P. Roy. Soc. B. 273: 13851389

Heffner EL, Sorrells ME, Jannink JL (2009) Genomic selection for crop improvement. Crop Sci. 49: 1-12

Hegde SG, Nason JD, Clegg JM, Ellstrand NC (2006) The evolution of California's wild radish has resulted in the extinction of its progenitors. Evolution 60: 1187-1197

Hooftman DAP, Oostermeijer JGB, Jacobs MMJ, den Nijs JCM (2005) Vital rates determine the performance advantage of crop-wild hybrids in Lettuce. J. Appl. Ecol. 42: 1086-1095 
Hooftman DAP, Oostermeijer JGB, den Nijs JCM (2006) Invasive behaviour of Lactuca serriola (Asteraceae) in The Netherlands: spatial distribution and ecological amplitude. Basic Appl. Ecol. 7: 507-519

Hooftman DAP, de Jong MJ, Oostermeijer JGB, den Nijs JCM (2007a) Modelling the long-term consequences of cropwild relative hybridization: a case study using four generations of hybrids. J. Appl. Ecol. 44: 1035-1045

Hooftman DAP, Nieuwenhuis BPS, Posthuma KI, Oostermeijer JGB, den Nijs JCM (2007b) Introgression potential of downy mildew resistance from lettuce to Lactuca serriola and its relevance for plant fitness. Basic Appl. Ecol. 8: $135-146$

Hooftman DAP, Oostermeijer JGB, Marquard E, den Nijs JCM (2008) Modelling the consequences of crop-wild relative gene flow: a sensitivity analysis of the effects of outcrossing rates and hybrid vigour breakdown in Lactuca. J. Appl. Ecol. 45: 1094-1103

Huxel GR (1999) Rapid displacement of native species by invasive species: effects of hybridization. Biol. Conserv. 89: 143152

Kirk H, Vrieling K, Klinkhamer PGL (2005) Maternal effects and heterosis influence the fitness of plant hybrids. New Phytol. 166: 685-694

Koopman WJM, Zevenbergen MJ, van der Berg RG (2001) Species relationships in Lactuca s.l. (Lactuceae, Asteraceae) inferred from AFLP fingerprints. Am. J. Bot. 88: 1881-1887

Lynch M, Walsh B (1998) Genetics and analysis of quantitative traits. Sunderland, Sinauer Associates, Inc.

Mercer KL, Andow DA, Wyse DL, Shaw RG (2007) Stress and domestication traits increase the relative fitness of cropwild hybrids in sunflower. Ecol. Lett. 10: 383-393

Milne RI, Abbott RJ (2000) Origin and evolution of invasive naturalised material of Rhodendron ponticum L. in the British Isles. Mol. Ecol. 9: 541-556

Moles AT, Gruber MAM, Bonser SP (2008) A new framework for predicting invasive plant species. J. Ecol. 96: 13-17
Orozco-ter Wengel P, Sørensen A, den Nijs JCM, Hooftman DAP (2005) Hybrids between lettuce and its wild relative have an increased likelihood of carrying crop specific genome segment. Abstracts of the 90th meeting of the Ecological Society of America, Montreal

Rapp RA, Wendel JF (2005) Epigenetics and plant evolution. New Phytol. 168: 81-91

Ridley CE, Ellstrand NC (2009) Evolution of enhanced reproduction in the hybrid-derived invasive, California wild radish (Raphanus sativus). Biol. Invasions 11: 2251-2264

Rieseberg LH, Raymond O, Rosenthal DM, Lai Z, Livingstone K, Nakazato T, Durphy JL, Schwarzbach AE, Donovan LA, Lexer C (2003) Major ecological transitions in wild sunflowers facilitated by hybridization. Science 301: 1211-1216

Ryder EJ (1999) Lettuce, Endive and Chicory. Wallingford, CAB International

Snow AA, Pilson D, Rieseberg LH, Paulsen MJ, Pleskac N, Reagon MR, Wolf DE, Selbo SM (2005) A B transgene reduces herbivory and enhances fecundity in wild sunflowers. Ecol. Appl. 13: 279-286

Snow AA, Culley TM, Campbell LG, Sweeney PM, Hegde SG, Ellstrand NC (2010) Long-term persistence of crop alleles in weedy populations of wild radish (Raphanus raphanistrum). New Phytol. 186: 537-548

Stewart CN, Halfhill MD, Warwick SI (2003) Transgene introgression from genetically modified crops to their wild relatives. Nat. Rev. Genet. 4: 806-817

Sweet JB (2009) The 10th International Symposium on the Biosafety of Genetically Modified Organisms (ISBGMO), Wellington, New Zealand, November 2008. Environ. Biosafety Res. 8: 161-181

Tutin TG, Heywood VH, Burges NA, Moore DM, Valentine DH, Walters SM, Webb DA (1976) Flora Europaea, vol. 4. Cambridge, Cambridge University Press

Vuilleumier S, Yearsley JM, Perrin N (2008) The fixation of locally beneficial alleles in a metapopulation. Genetics $\mathbf{1 7 8}$ : $467-475$ 